\title{
Improvement of systematic uncertainties for the neutron lifetime experiment at J-PARC
}

T. Mogi, ${ }^{a, *}$ T. Hasegawa, ${ }^{b}$ K. Hirota, ${ }^{b}$ G. Ichikawa, ${ }^{c}$ S. Ieki, ${ }^{d}$ T. Ino, ${ }^{c}$ Y. Iwashita, ${ }^{e}$

S. Kajiwara, ${ }^{a}$ Y. Kato, ${ }^{a}$ M. Kitaguchi, ${ }^{f}$ R. Kitahara, ${ }^{g}$ J. Koga, ${ }^{h}$ S. Makise,${ }^{h}$

S. Matsuzaki, ${ }^{h}$ K. Mishima, ${ }^{c}$ K. Morikawa, ${ }^{b}$ N. Nagakura, ${ }^{a}$ H. Okabe, ${ }^{b}$ H. Otono, ${ }^{i}$ Y. Seki, ${ }^{j}$ D. Sekiba, ${ }^{k}$ T. Shima, ${ }^{l}$ H. E. Shimizu, ${ }^{m}$ H. M. Shimizu, ${ }^{b}$ Y. Sugisawa, ${ }^{k}$

N. Sumi ${ }^{c}$ H. Sumino, ${ }^{n}$ T. Tanabe,${ }^{c}$ T. Tomita, ${ }^{h}$ H. Uehara, ${ }^{h}$ T. Yamada, ${ }^{a}$

S. Yamashita, ${ }^{o}$ K. Yano ${ }^{h}$ and T. Yoshioka ${ }^{i}$

${ }^{a}$ Department of Physics, Graduate School of Science, The University of Tokyo, Tokyo, 113-0033, Japan

${ }^{b}$ Department of Physics, Graduate School of Science, Nagoya University, Nagoya, 464-8602, Japan

${ }^{c}$ KEK, High Energy Accelerator Research Organization, Tsukuba, 305-0801, Japan

${ }^{d}$ Research Centre for Neutrino Science, Tohoku University, Miyagi, 980-8578, Japan

${ }^{e}$ Institute for Integrated Radiation and Nuclear Science, Kyoto University, Kumatori, 590-0494, Japan

${ }^{f}$ Kobayashi-Maskawa Institute for the Origin of Particles and the Universe (KMI), Nagoya University, Nagoya, 464-8602, Japan

${ }^{g}$ Department of Physics, Graduate School of Science, Kyoto University, Kyoto, 606-8502, Japan

${ }^{h}$ Department of Physics, Graduate School of Science, Kyushu University, Fukuoka, 819-0395, Japan

${ }^{i}$ Research Center for Advanced Particle Physics (RCAPP), Kyushu University, Fukuoka, 819-0395, Japan

${ }^{j}$ Center for Physics and Mathematics, Osaka Electro-Communication University, Osaka, 572-8530, Japan

${ }^{k}$ Institute of Applied Physics, University of Tsukuba, Tsukuba, 305-8573, Japan

${ }^{l}$ Research Center for Nuclear Physics (RCNP), Osaka University, Ibaraki, 567-0047, Japan

${ }^{m}$ School of High Energy Accelerator Science, The Graduate University for Advanced Studies, Tsukuba, 305-0801, Japan

${ }^{n}$ Department of General Systems Studies, Graduate School of Arts and Sciences, The University of Tokyo, Tokyo, 153-8902, Japan

o International Center for the Elementary Particle Physics (ICEPP), The University of Tokyo, Tokyo, 113-0033, Japan

E-mail: mogi@icepp.s.u-tokyo.ac.jp

The neutron lifetime is one of the important parameters for particle physics and astrophysics. There is a $4.6 \sigma(9.5 \mathrm{~s})$ discrepancy between the results of the two typical methods. To solve this discrepancy, a new neutron lifetime experiment with a different method is in progress at J-PARC, and this experiment published the first result in 2020 of $898 \pm 10$ (stat.) $+15 /-18$ (syst.) s. We have performed improvements to reduce systematic uncertainties towards $1 \mathrm{~s}$ accuracy. The methods to measure the amount of ${ }^{3} \mathrm{He}$ injected and in the working gas have been upgraded, and a new model for the space charge effect in the Monte Carlo simulation has been implemented.

*** Particles and Nuclei International Conference - PANIC2021 ***

*** 5 - 10 September, $2021 * * *$

$* * *$ Online $* * *$

${ }^{*}$ Speaker 


\section{Introduction}

A free neutron decays into a proton, an electron, and an antineutrino in a lifetime of about $880 \mathrm{~s}$. The neutron lifetime dominates the uncertainty on ${ }^{4} \mathrm{He}$ abundance in the Big Bang Nucleosynthesis, and it also determines $V_{u d}$ term in the Cabibbo-Kobayashi-Maskawa quark mixing matrix. Although the neutron lifetime is very important in modern physics, there is a $4.6 \sigma$ ( $9.5 \mathrm{~s}$ ) discrepancy between the results of the two typical methods: the beam method $(888.0 \pm 2.0 \mathrm{~s})$ and the storage method $(878.4 \pm 0.5 \mathrm{~s})[1,2]$. The beam method measures the neutron flux and decay products by different detectors, and the storage method counts survival neutrons after some storage times. This discrepancy is called the neutron lifetime puzzle and is unsettled.

\section{Neutron lifetime experiment at J-PARC}

A new neutron lifetime experiment is in progress at J-PARC MLF BL05. In this method, the neutron flux and decay electrons are measured simultaneously by a time projection chamber (TPC) filled with a working gas mixture of ${ }^{4} \mathrm{He}$ of $85 \mathrm{kPa}$ and $\mathrm{CO}_{2}$ of $15 \mathrm{kPa}$, and ${ }^{3} \mathrm{He}$ gas to evaluate the neutron flux. The systematic uncertainties are different from the previous beam method because we measure the decay electrons, whereas the previous beam experiments measured decay protons. The pulsed neutron beam is divided into 5 bunches by the spin flip chopper (SFC), which consists of flipper coils and magnetic supper mirrors, and is injected into the TPC. Forming short neutron bunches about $40 \mathrm{~cm}$, the interactions between neutrons and wall materials in the TPC can be significantly reduced by a time-of-flight selection. The TPC consists of the multi-wire proportional chamber (MWPC), a drift plate, and ${ }^{6} \mathrm{LiF}$ internal walls. Then the neutron lifetime is determined as

$$
\tau_{n}=\frac{1}{\rho \sigma_{0} v_{0}} \frac{\left(S_{3_{\mathrm{He}}} / \varepsilon_{3 \mathrm{He}}\right)}{\left(S_{\beta} / \varepsilon_{\beta}\right)},
$$

where $\rho$ is the number density of ${ }^{3} \mathrm{He}$ nuclei, $\sigma_{0}=5333 \pm 7$ barn is the cross-section of ${ }^{3} \mathrm{He}(n, p){ }^{3} \mathrm{H}$ reaction at the neutron velocity $v_{0}=2200 \mathrm{~m} / \mathrm{s}, S_{3^{H e}}$ and $S_{\beta}$ are the numbers of measured events of ${ }^{3} \mathrm{He}(n, p){ }^{3} \mathrm{H}$ reactions and the $\beta$ decays respectively, and $\varepsilon_{3} \mathrm{He}$ and $\varepsilon_{\beta}$ are the signal selection efficiency determined by Monte Carlo (MC) simulation. The first result of this experiment was published in 2020 [3]:

$$
\left.\tau_{n}=898 \pm 10 \text { (stat. }\right)+15 /-18 \text { (syst.) s. }
$$

\section{Improvement of systematic uncertainties}

We have performed the upgrades of the measurement environment and MC simulation to reduce systematic uncertainties towards $1 \mathrm{~s}(0.1 \%)$ accuracy.

\subsection{The number density of ${ }^{3} \mathrm{He}$ nuclei}

The TPC is in a vacuum chamber filled with a working gas mixture of $\mathrm{He}$ and $\mathrm{CO}_{2}$ of $100 \mathrm{kPa}$. To measure the neutron flux, ${ }^{3} \mathrm{He}$ gas with a pressure of about $100 \mathrm{mPa}$ is admixed in the chamber, and the chamber is sealed to keep the number density constant. The total number density of ${ }^{3} \mathrm{He}$, $\rho$, is the sum of two components:

$$
\rho=\rho_{\text {inject }}+\rho_{\text {workgas }},
$$


where $\rho_{\text {inject }}$ is the gas amount injected from a ${ }^{3} \mathrm{He}$ gas cylinder, and $\rho_{\text {workgas }}$ is the small ${ }^{3} \mathrm{He}$ amount of about $10 \mathrm{mPa}$ in the commercial He.

To inject ${ }^{3} \mathrm{He}$ gas of about $100 \mathrm{mPa}$ into the vacuum chamber with high accuracy, the ${ }^{3} \mathrm{He}$ gas is injected into a small reference volume with high pressure of about $3 \mathrm{kPa}$, then released into the chamber. The volume ratio of the reference volume to the vacuum chamber is necessary to be measured with high accuracy since it also determines the accuracy of $\rho_{\text {inject }}$. The volume ratio was calculated by the pressure change when $\mathrm{He}$ gas is diffused from the small volume to the vacuum chamber. Due to the dynamic range of the pressure gauge, the measurement was done in several steps. A new type of transducer with a wide dynamic range and a $300 \mathrm{~cm}^{3}$ larger bottle were introduced to obtain a more accurate volume ratio. The volume ratio was determined as $(2.0145 \pm 0.0013) \times 10^{3}$, whereas the ratio was equivalent to $(2.0159 \pm 0.0035) \times 10^{3}$ in the previous measurement. The resulting accuracy of $\rho_{\text {inject }}$ is about $0.1 \%$.

The measurement accuracy of $\rho_{\text {workgas }}$ by a conventional method, mass spectrometry, was $1-2 \%$. We developed a new method to measure the number density of ${ }^{3} \mathrm{He}$ in the commercial $\mathrm{He}$ using $\mathrm{N}_{2}$ gas with neutron beam in the TPC. Then the density is calculated as

$$
\rho_{\text {workgas }}=\frac{S_{3^{H e}} / \varepsilon_{3} \mathrm{He}}{S_{{ }^{4} \mathrm{~N}} / \varepsilon_{14} \mathrm{~N}} \frac{\sigma_{14}}{\sigma^{3} \mathrm{He}} \rho_{\mathrm{N}_{2}}
$$

where $S_{3} \mathrm{He}$ and $S_{{ }^{4} \mathrm{~N}}$ are the number of measured events of ${ }^{3} \mathrm{He}(n, p){ }^{3} \mathrm{H}$ reactions and ${ }^{14} \mathrm{~N}(n, p){ }^{14} \mathrm{C}$ reactions respectively, $\sigma_{3} \mathrm{He}$ and $\sigma_{14} \mathrm{~N}$ are their capture cross-sections, and $\rho_{\mathrm{N}_{2}}$ is the number density of nitrogen gas. We have performed the measurement of $\sigma_{14} \mathrm{~N}$ with high accuracy $(1.868 \pm$ 0.007 barn) [4]. The results of the two methods were consistent, and the uncertainty of $\rho_{\text {workgas }}$ by the $\mathrm{N}_{2}$ method achieved to $0.4 \%$ as shown in Table 1 .

Table 1: The ${ }^{3} \mathrm{He}$ amount in the commercial He cylinders.

\begin{tabular}{c|c|c}
\hline & ${ }^{3} \mathrm{He} /{ }^{4} \mathrm{He}$ ratio of cylinder \#1 & ${ }^{3} \mathrm{He} /{ }^{4} \mathrm{He}$ ratio of cylinder \#2 \\
\hline Mass spectrometry & $0.0919 \pm 0.0040 \mathrm{ppm}$ & $0.0908 \pm 0.0016 \mathrm{ppm}$ \\
$N_{2}$ method & $0.0918 \pm 0.0006 \mathrm{ppm}$ & $0.0920 \pm 0.0004 \mathrm{ppm}$ \\
\hline
\end{tabular}

\subsection{Gain reduction model in MC simulation}

In wire chambers, the gas multiplication factor is reduced by positive ion clusters around the anode wires generated by primary and secondary electrons. This effect is known as the space charge effect, and the effect is implemented in the MC simulation as a gain reduction factor $s$ [5]:

$$
s=\frac{\log \left(1+f \frac{d E}{d l} G_{0}\right)}{f \frac{d E}{d l} G_{0}}
$$

where $f$ is a fitting parameter, $G_{0}$ is the multiplication factor without gain reduction, and $d E / d l$ is an energy deposit per unit length along the anode wire. The current model causes a difference between the measured energy deposit spectrum and that of MC as shown in the left side of Fig. 1. The difference results in the uncertainty of the efficiency of $\beta$ decays, $\varepsilon_{\beta}$, and the value is $0.3-1.0 \%$. 
To reduce the difference, a new model was implemented to the factor $s$. In the previous computation, the factor $s$ was calculated by the number of electrons in a mesh divided into 2-6 $\mathrm{mm}$ sections along the anode wires. We newly introduced the contribution of electrons dispersed by a Gaussian distribution from the neighboring meshes. The parameters $f$ and $G_{0}$ have also been refined. As a result, the new computational model has improved the reproducibility of the energy deposit spectrum as shown in the right side of Fig. 1, and the uncertainty is reduced to $0.04-0.8 \%$.
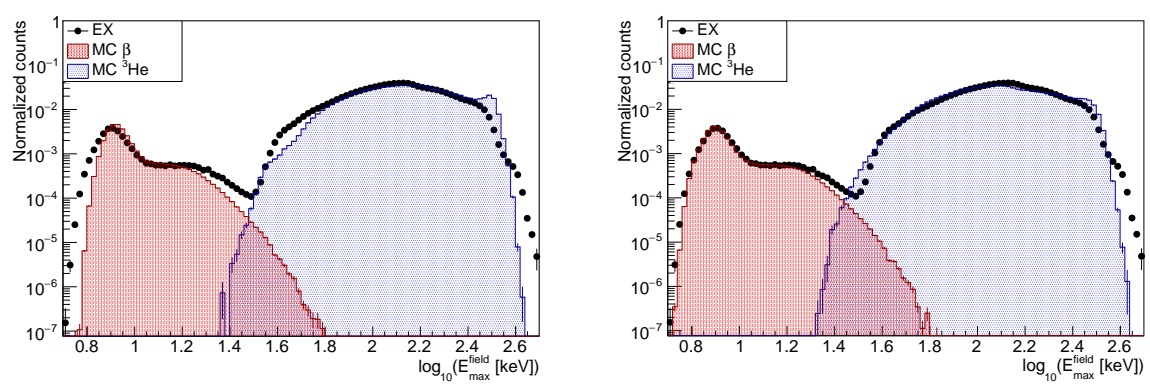

Figure 1: Energy deposit spectra of previous MC (left) and improved MC (right). The red and blue hatches represent the energy deposit distribution of $\beta$ decay events and ${ }^{3} \mathrm{He}(n, p)^{3} \mathrm{H}$ events, respectively. The black dots show the measured energy spectrum.

\section{Conclusion}

A new experiment to solve the neutron lifetime puzzle is in progress at J-PARC. We have performed improvements to reduce systematic uncertainties towards $1 \mathrm{~s}$ accuracy. To improve the measurement accuracy of the number density of ${ }^{3} \mathrm{He}$ injected and in the working gas, new methods to determine the volume ratio and to measure ${ }^{3} \mathrm{He}$ amount in the commercial $\mathrm{He}$ are developed. A new model to calculate the space charge effect in the MC simulation has also been implemented. By these improvements, an accuracy of $1 \mathrm{~s}$ has been achieved for several of the systematic uncertainties.

\section{References}

[1] P.A. Zyla, et al. (Paticle Data Group), The review of particle physics, Prog. Teor. Exp. Phys., 2020, 083 C01 (2020).

[2] F. M. Gonzalez, et al., Improved Neutron Lifetime Measurement with UCN $\tau$, Phys. Rev. Lett., 127, 162501 (2021).

[3] K. Hirota, et al., Neutron lifetime measurement with pulsed cold neutrons, Prog. Teor. Exp. Phys., 2020, $123 \mathrm{C} 02$ (2020).

[4] R. Kitahara, et al., Improved determination of thermal cross section of ${ }^{14} N(n, p){ }^{14} C$ for the neutron lifetime measurement, Prog. Teor. Exp. Phys., 2019, 093 C01 (2019).

[5] Y. Arimoto, et al., Development of time projection chamber for precise neutron lifetime measurement using pulsed cold neutron beams, Nucl. Instrum. Methods Phys. Res. A, 799, 187-196 (2015). 